\title{
Physico-chemical factors conditioning the electronic conduction of conductive polymers
}

\author{
Fatiha SAOUTI ${ }^{1}{ }^{10}$,Soufiyan HAMHAM ${ }^{1}$, Abdelouahed CHARQAOUI ${ }^{1}{ }^{\circ b}$ and Youssef NAIMI ${ }^{1}{ }^{10}$ \\ ${ }^{I}$ Faculty of science BEN M'SIK, University Hassan II of Casablanca, Department of chemistry, Casablanca, Morocco \\ Saoutifatiha2@gmail.com
}

Keywords: Physicochemical, electrochemical, Gap energy, cyclic voltammetry, polyaniline.

Abstract: The driving idea and the main objective of this synthetic work is the study of physicochemical factors such
as the dielectric constant of the solvent, the polarizability of the chains and the Gap energy of the
conductive polymers, these characteristics condition their properties. electrical and electrochemical.
We focused our study on polyaniline, two synthetic methods are presented: chemical polymerization and
electropolymerization with emphasis on the theoretical aspects of the phenomena involved as well
as on the electrochemical methods used such as cyclic voltammetry. We have therefore developed with
maximum clarity the links existing between the physico-chemical properties of polyaniline and its electrical
conductivity.

*Corresponding author:saoutifatiha2@ gmail.com 


\section{INTRODUCTION}

Conductive polymers have been the subject of increased attention from the scientific community in recent decades, due to their vast field of technological applications which is becoming increasingly wide, especially in the fields of molecular electronics. , excitonic and optronic, as well as in organic electrochemistry and catalysis.

Understanding the intimate links between the electronic structure and the stereochemistry of organic macromolecules with semiconductor or metallic conductivity due to the delocalization of electrons from the $\pi$ molecular orbitals along the macromolecular chains, will therefore favor the explanation and apprehension of their electrical, electrochemical and optical properties as well as the different conduction modes, and will also allow a better explanation of these properties especially in the development of ultra-thin conductive or semiconductive layers by chemical polymerization or by electrodeposition; these structures are used in the development of the immobilizing surfaces of DNAs, RNAs or proteins in amperometric or impedimetric biosensors (Bioelectronics), as well as OJI ("Organic" junction transistor), Oleds (organic lightemitting diode), nanoelectrodes for nanoelectrochemistry, semiconductor electrochemistry and photo-electrochemistry also their multitudes of applications in digital display, protection against corrosion, quantum dots (nanodots) and organic photovoltaic cells (OPVC).

Much more confusing and much more spectacular applications are the subject of intensive research such as the bipolaronic and excitonic condensates "The excitonic insulating state" which will find wide applications in quantum cryptography and computing, as well as fractional quantum fluids (Spin separation -charge) and skyrmionic computers using carbon foam, and paramagnetic polymers and annular diamagnetic currents of aromatic polymers.

\section{Conductive Polymers: General Definition}

In recent years, a completely new and effervescent field of the physical chemistry of materials has arisen, it is the electrochemistry and electronics of polymers with very interesting electrical conductivity and semi-conductivity. which gives them electrical, electrochemical and optical properties due to their low ionization potential and their high electronic affinity.

These extraordinary properties are explained by the existence of conjugation in these polymer chains, that is to say the alternation of molecular orbitals $p$ resulting from the lateral overlap of the atomic orbitals $\mathrm{p}$, and molecular orbitals $\mathrm{s}$ due to the axial overlap of atomic orbitals s. due to the high mobility of the $\pi$ electrons there is a strong delocalization of the molecular orbitals of the polymers, i.e. the presence of almost free electrons which can move along the polymer chain, this movement cannot $\mathrm{s}$ " effect that by electron jump from the high energy occupied molecular orbital to the vacant low energy molecular orbital, this is the transition $\pi-\pi *$. The doped conductive polymers have an intrinsic electronic conductivity ranging from about $10^{-4}$ to $10^{2} \mathrm{~S} \mathrm{~cm}-1$ due to the extension of the doped state[1].

In the neutral state (undoped), these materials are only semiconductors and the electronic conductivity appears only when the material is doped with ions of small size (great polarizing power) in addition the conductive polymers present interesting mechanical properties such as lightness, flexibility, meshability and hardness (carbon nanotubes and graphene).

This type of doping is usually carried out by chemical or electrochemical oxidation of the monomer, in which the polymer chains lose electrons which gives them a positive charge, the electrical neutrality of the material obtained is ensured by the incorporation of small counter-ions to from the electrolytic solution [2]. Conductive polymers have become more and more interesting and are currently the subject of increased attention due to the range of their applications in different fields, light-emitting diodes, Schottky diodes, tunnel diodes, biosensors, catalysis, ionoselective membranes, field effect transistors, photovoltaic cells, digital display, corrosion protection agents, smart windows, RAMs, nano-dotes, nano-wires, biomaterials (nano-mites), bipolaronic and excitonic computers ....

The functionalization of conductive polymers is ensured by the specificity of the dopant used, this makes it possible to control its physico-chemical properties, there are several types of doping such as chemical, electrochemical and optical doping. It is also possible to use crosslinking techniques, ion trapping, physisorption and covalent coupling to control the electrical conductivity of the polymer. Therefore the electronic and steric structure of the polymer as well as its interactions with the dopant constitute the determining factors of its electrical and optical properties [3].

In 1957 Natta observed a high electrical conductivity of polyacetylene treated with a halogen. Many other conjugated polymers therefore showed a transition from the insulating state to a highly conductive state, such as polyanniline, polythiophene, polypyrrole and polyphenylenevinylene. 
By the diversity of the electrical conduction mechanisms of conductive polymers ranging from metallic conductivity by electrons, semiconductor by electrons and holes, ionic conductivity by ions and ion vacancies, conductivity by Pieirls distortion and soliton effect and finally conductivities by hopping effect and polaronic and bipolaronic conductivities, as well as their physico-chemical and electrochemical properties. Conductive polymers then present a new field of electronics which is molecular electronics (polaronic, bipolaronic, solitonic, eccentric and molecular magnonic as well as the spin-charge separation effects of the electron, in a solid such as graphene for example the electron behaves as if it were devoid of mass, like a chiral particle made up of three particles the spinon, the chargeon and the orbiton).

Among the most interesting polymers by their rich applications, those which are illustrated below:

- Polypyrrole (PPy)

- Polyanilin (PANI)

- Poly(3,4-ethylenedioxythiophene)(PEDOT)

- Polythiophene (PTh)

- Poly (p-phenylene) (PPP)

- Poly-p-phenylene-sulphide (PPS)

- Poly (p-phenylenevinylene) (PPV)

- Polyacetylene (PAc)

- Polyazulene (PAZ)

- Polybutadiene (PBD)

The remarkable property of these molecules is the fact that it combines the electropositive character of metals and the physico-chemical properties of polymers, the major interest it is related to the electrical and optical properties of its compounds with their electronic structures, their stereochemistry and their redox powers [4].

The first interesting facts that have been observed for conductive polymers is the remarkable increase in their electrical conductivity when they undergo physicochemical modifications (the oxidation of polyacetylene by odine vapor increases its conductivity by 10 million of times) [5,6].

The difficulty of synthesis of polyacetylene as well as its instability in the air limited its use for technological applications, it is for this reason that the quest for an optimal conductive polymer began. Polyheterocyclic macromolecules then emerged as a family of full-fledged PCs which are physicochemical stable and which are good conductors, having a high conductivity / mass ratio and which can be used as biomaterials (prostheses, artificial organs, pacemaker, heart valve drainage, biodegradable paramagnetic and magnetic markers) by making them biocompatible, biodegradable (harmless to the body and the environment) and porous (highly selective membranes) [7-9].

The field of biosensors, i.e. devices capable of measuring the biological and physiological parameters of a living organism (DNA biochips, amino acid networks, bioFet, enFet, immunosensors, glucose sensors ...). the surface structure and morphology as well as the conductivity of the synthesized thin layers constitute two crucial points for the implementation of sensors and biosensors. the porosity and the uniformity of the surface morphology in addition to the superior conductivity allow a great exploitation of conductive polymers in this sector of technology. These measurement, detection and assay systems are based on the substantial modification of the optical and electrical properties of these polymers such as undoped and doped polyacetylene, polypyrrole, polyaniline and polythiophene have shown a semi-conductivity which can be used in the manufacture of such devices.

\section{Polyaniline}

Polyaniline ranks first among conductive polymers, it is a polymer constructed from phenyl belonging to the chemically flexible $\mathrm{NH}$ series of the polymer linked by a loop on two sides [10].

By a redox process the electrical conductivity of the PANI allows to pass from one form to another, the degree of its protonation is a crucial factor for the conductivity of the emeraldine form of polyaniline. (The electrical conductivity increases by ten orders of size when its protonation increases by 20\%) [11].

Polyaniline represents the material of choice for contemporary and future electronic technology thanks to its easy production, its electrical and electronic properties which are controllable and reversible by oxidation and protonation (non-redox doping)[12].

PANI has the general formula [(- B - NH B $\mathrm{NH}) \mathrm{n}\left(\mathrm{B} \mathrm{N}=\mathrm{Q}=\mathrm{N}_{-}-1-\mathrm{n}\right] \mathrm{m}$ in which the band $\mathrm{Q}$ designates the rings in the benzenoid and quinonoid forms, respectively.

It exists in three oxidation states, leucoemeraldine $(\mathrm{n}=1)$ fully reduced form, pernigraniline $(\mathrm{n}=0)$ fully oxidized form, these two forms have low electrical conductivity, on the other hand emeraldine corresponding to the partially oxidized $(\mathrm{n}=1 / 2)$ has interesting electrical properties due to its presence in two forms, insoluble conductive salt of green color which can be doped with ammonium hydroxide to give the soluble basic form of blue color [ 13]. 
In order to remedy this difficulty, functionalized dopants have been used to improve the solubility of polyaniline in particular and the conjugated polymers in general[14-16].

Several synthetic methods based on the oxidation of aniline have been proposed for the development of polyaniline, among these techniques include electrochemical polymerization [17], oxidative chemical polymerization [18], photolysis [19], radiolysis [20,21], polymerization initiated by photochemical way as well as polymerization on adsorbent surface and enzymatic polymerization. The most used techniques are chemical and electrochemical polymerizations, the latter is carried out in aqueous electrolytes of strong acids (sulfuric acid, hydrochloric acid ...) according to a mechanism which results in the formation of an anilinium cation radical by oxidation of 1 'aniline on the electrode [22].

Thanks to the virtues in the electrical properties of polyaniline, it can be considered as a hybrid with an electrically conductive biopolymer for especially electrochemical biosensors as well as a redox biomaterial for biomedical applications, or as a matrix for the preparation of nanocomposites, nanoparticles and nanopaillettes based on conductive polymers and especially polyaniline using the techniques offered by nanotechnology (electroplating, spraying by ion trapping, grinding, sol-gel transformation, micro and nano dissolution, micro and nano dispersion, discharges by electric arc, plasma discharges) [23-31].

Polyaniline can also be used by virtue of its stability in the development of electrochemical nanocells [32, 33], and photovoltaic and optoelectronic devices [34], EMI shielding [35].

\section{Conductivity of the conductive polymers}

The conductivity in organic polymers represents a physico-chemical phenomenon which has attracted attention since the 1970s with the work of Rose, Heeger and Macdiarmid. His research focused on the different methods of synthesis of conductive and semi-conductive polymers as well as the links between their electronic structure and their stereochemistry and their optoelectric properties. Several synthetic methods have been proposed such as electropolymerization, ultrasound assisted polymerization, interfacial polymerization, photopolymerization and chemical polymerization.

We will focus on electropolymerization because of its flexibility and ease of implementation as well as the possibility of control and monitoring of the reaction by electrochemical methods available on the one hand and not too laborious on the other On the other hand, the methods of choice that can be used in electropolymerization are: polarography, anodic redissolving voltammetry, cyclic voltammetry, impedance spectroscopy and transitometry.

These techniques allow us to measure the redox potential of the polymer formed, therefore its Homo (High Occuped Molecular Orbital) and Lumo (Low Unoccuped Molecular Orbital) energies, to determine the formation constant, the degree of polymerization and the rate constants of the polymer formation reactions and finally the dosage of the quantity of the polymer formed and sometimes even the statistical distribution of molecular weights.

In the case of the polymerization of aniline into polyaniline, there is an oxidation of the aniline as well as the formation of imine and amine bonds, this oxidation requires a support electrolyte $\left(\mathrm{H}_{3} \mathrm{O}^{+}, \mathrm{SO}_{2}\right.$ ${ }^{4}$ ), the $\mathrm{H}_{3} \mathrm{O}^{+}$behave like catalysts, the strong electric field created by the $\mathrm{H}_{3} \mathrm{O}^{+}$leads to the polarization of the aniline and the deformation of its electronic cloud, which therefore leads to a transfer of electrons from the aniline to the anode opposite -vis the electrolyte-electrode interface. the interaction image force between the surface charge of the electrode and the oxidized aniline molecules cause their desolvation and their adsorption on the surface of the electrode and finally the coulomb barrier created by the electrode due to the cloud electronics of the crystal lattice of the electrode as well as the electrons transferred result in an attraction between the aniline molecules oxidized in spite of their positive charges and because of the small difference between the HOMO and LUMO energies of the aniline, the term of 1 orbital interaction is preponderant compared to electrostatic interactions and steric interactions and this leads to the formation of imine and amine molecular orbitals between the quinoid cycles of aniline therefore the electropolymerization and the electrodeposition of aniline on the anode form of polyaniline. To prepare thin layers of polyaniline, we opt for non-polar solvents so as not to dissolve the polyanline. The solvents of choice used are: carbon tetrachloride, chlorobenzene, tetrahydrofuran, carbon sulfide, etc. These solvents provide by their weak dielectric constants and their weak dipole moments the selfassembly of the polymer chains in a fibrillar structure in supramolecular organization further promoting their conductivity and the mobility of the charge carriers as well as the regiregularity of the polymer, that is to say a periodic or quasi-periodic structure creating thus potentials which strikingly remind us of the periodic Bloch potentials of the ordinary crystal lattice. Interchain interactions must be predominant over chain-solvent interactions 
therefore this requires the use of solvents of very low polarities or apolar, all these interactions whether inter-chain or chain-solvent are due to dipole-dipole interactions, interactions of van der waals of dipole-quadrupole interactions......

Mathematically we can identify the links between the length of the polymer chain, the polarizability of the polymer its dipole moment and the mobility of the charge carriers therefore the conductivity of the polymer. If we assimilate the macromolecular chain to a fiber of great axial ratio, that is to say of a radius negligible compared to its length, under these conditions we express the polarizability of the chain by the following equation:

$$
\alpha=\left(\frac{\varepsilon_{\text {red }}-1}{\varepsilon_{\text {red }}+2}\right) \pi \mathrm{r}^{2}\langle\mathrm{~L}\rangle
$$

Where $r$ is the geometric radius of the chain

$\langle\mathrm{L}\rangle$ the average length of the chain

$\varepsilon_{\text {red }}$ The reduced electric constant which accounts for the weak interactions between the polymer chains and the solvent and it is expressed as follows:

$$
\varepsilon_{\text {red }}=\frac{\varepsilon_{\text {solv }} \varepsilon_{\text {poly }}}{\varepsilon_{\text {solv }}+\varepsilon_{\text {poly }}}
$$

$\varepsilon_{\text {solv }}$ is the dielectric constant of the solvent

$\varepsilon_{\text {poly }}$ is the dielectric constant of the polymer

The average length of the chain is linked to its molecular mass:The average length of the chain is linked to its molecular mass:

$$
\langle\mathrm{L}\rangle=\frac{\langle\dot{\mathrm{M}}\rangle}{\pi \mathrm{r}^{2} \rho \mathrm{N}_{\mathrm{A}}}
$$

Where $\rho$ : the density of the polymer

Is there a relationship between the average length of the chain and therefore its average molecular weight and the mobility of charge carriers?

Mobility is an increasing function of the length of the chain, that is to say:

$$
\mu=\frac{\langle L\rangle^{2}}{\tau_{\text {jump }} V_{\text {int }}}
$$

Where $\tau_{\text {jump }}$ is the jump time of the electrons through the $\pi$ orbitals.

$\mathrm{V}_{\text {int }}$ designates the internal potential created by the chain itself and which has nothing to do with the interfacial electric field generated by the concentration gradients of the charge carriers at the electrode-polymer interface.

Let us explain in the expression of mobility as a function of the average molecular mass:

$$
\mu=\frac{\langle\mathrm{M}\rangle^{2}}{\pi^{2} \mathrm{r}^{4} \rho^{2} \mathrm{~N}_{\mathrm{A}}{ }^{2} \tau_{\text {jumP }} V_{\text {int }}}
$$

High molecular weight polymers will therefore be more conductive than low molecular weight polymers.
We show that the internal electric potential is an increasing function of the polarizability and the mean dipole moment of the chain and inversely proportional to the inter-chain distance $\delta$ so that:

$$
V_{\text {interne }}=\frac{\alpha \mu_{\text {dip }}}{4 \pi \varepsilon \delta^{5}}
$$

For an electrically charged chain, the internal potential is expressed by:

$$
\mathrm{V}_{\text {interne }}=\frac{\alpha\langle\mathrm{q}\rangle}{4 \pi \varepsilon \delta^{4}}
$$

In the case of a non-polar chain the electric field created by a neighboring chain leads to polarization therefore from the first the interaction energy can be given by:

$$
\Delta \mathrm{U}_{\text {interaction }}=\frac{-\alpha^{\prime} \mathrm{V}_{\text {interne }}{ }^{2}}{2 \delta^{2}}
$$

Substitute the internal potential by its expression found above, we have:

$$
\Delta \mathrm{U}_{\text {interaction }}=\frac{-\alpha^{\prime} \alpha^{2} \mu_{\mathrm{dip}}^{2}}{32 \pi^{2} \varepsilon^{2} \delta^{12}}
$$

We note that the inter-chain interaction energy decreases very quickly with the distance in absolute value, this is why we must opt for solvents with very low dielectric constant.

For an electronic or hole conductivity, the conductivity is expressed as a function of the mobility of the charge carriers by the following formula:

$$
\sigma=\mu \mathrm{q} \quad 10
$$

where $\mathrm{n}$ is the density of the charge carriers and $\mathrm{q}$ the elementary charge.

So we can propose the expression below which relates the electrical conductivity with the physicochemical parameters of the polymers.

$$
\sigma=\frac{16 \mathrm{q}\langle\mathrm{M}\rangle^{2} \mathrm{n}}{\mathrm{N}_{\mathrm{A}} \mathrm{r}^{4} \rho^{2} \tau_{\text {jump }}} \cdot\left(\frac{\varepsilon \delta^{5}}{\alpha \mu_{\text {dip }}}\right)^{2}
$$

And we can even link the energy of the interchain interaction for two identical chains with the oxidation potential of the polymer.

$$
\mathrm{U}_{\text {int }}=\frac{-\alpha^{2} \mathrm{q}}{12 \pi \varepsilon \delta^{6}}\left|\mathrm{E}_{\mathrm{ox}}\right|
$$

So we can easily by means of electropolymerization connect the structural parameters of the polymer with the redox potentials translating the affinity of the macromolecule to electrons, the gap energy of the polymer is equal to:

$$
\mathrm{Eg}=\mathrm{q}\left|\mathrm{E}_{\text {red }}^{\mathrm{s}}-\mathrm{E}_{\mathrm{ox}}^{\mathrm{s}}\right|
$$

This relationship is very important because it makes it possible to elucidate the relationships 
between the spectroscopic and electrochemical quantities, that is to say the coupling between the electrochemical and spectroscopic methods for the characterization of conductive polymers, this is spectroelectrochemistry (infrared spectroscopy coupled with cyclic voltammetry, RAMAN spectroscopy coupled with cyclic voltammetry either with impedancemetry or with chronopotentiometry, polarography coupled with RPE etc....).

The conductivity of polymers is due in the first rank to conjugation, that is to say the existence of an alternation between the orbitals $\sigma$ which constitute the skeleton of the polymer chain, and the orbitals $\pi$ where the electrons are very labile and they can delocalize along the macromolecule it is the first cause of the macromolecular conductivity. During the transition of the electrons from the stable $\pi$ orbitals to the unstable $\pi *$ orbitals, they leave vacant orbitals behind them which represent the holes, this is the macromolecular semiconductor. We can relate the mobility and the conductivity to the coordination of the polymer chains as well as the energies of the orbitals $\pi$ and $\pi *$ (HOMO - LUMO).

Let us now leave aside this point for a further development and approach another mode of conduction in polymers, because of the existence of aromatic cycles, the diamagnetic annular electronic currents provide these macromolecules like polyaniline for example an electronic conductivity due to electric currents generated by the electrons of the quinoid cycles verifying Huckel's rule (mesomeric effect): $\mathrm{N}=4 \mathrm{n}+2$ ( $\mathrm{N}$ : number of delocalized electrons / $\mathrm{n}$ : number of aromatic cycles).

Polaronic and bipolaronic conductions also exist in conductive polymers, a polaron is a kind of entity constituted by an electron or a hole trapped by potential wells created by the folds, the windings and the twists of the macromolecular chains by inductive effects and electromers, these positive and negative polarons can propagate along the macromolecule and the polaronic conductivity increases more and more with the regoregularity of the conductive material due to the organization and the structuring of the polymer chains in fibrillar, quasi-crystalline, crystalline configurations. superhelical...

Bipolarons are structures due to interactions and coupling between polarons, each bipolaron can be made up either of two positive polarons, or of two negative polarons interacting with intra-chain vibrations, i.e. the exchange of phonons thus creating an attractive force surpassing the electrostatic repelling force. This bipolar conductivity can constitute the starting point towards the theoretical explanation of high temperature superconductivity which is not framed by the BCS theory (Bardeen, Coopper, Schreiffer), the neutral bipolarons which do not contribute to the conductivity can low temperature form new phases of quantum liquids.

Another mode of conduction can also be considered, it is the inter-chain conduction by $\pi \pi$ interaction, by electron transfer by tunnel effect through hydrogen bonds (polyurethanes, polypyrroles, polyamines, polypeptides, polynucleotides) or sometimes through halogenated bonds.

The distortion and the break in symmetry due to the degeneration lift lead to the formation of solitons that is to say an excitation propagating in a nonlinear and dispersive medium, these solitons can in turn associate in bisolitons ensuring a other mode of conduction in organic conductive and semiconductor polymers (SSH Shear, Schreiffer, Heeger model).

\section{Electrochemical polymerization of conductive polymers}

-Determination of the plating speed:

Under FARADAY's law the amount of electricity flowing in the solution can be expressed as a function of the current intensity imposed by the following relationship:

$$
Q=\int_{t 1}^{t 2} I \cdot d t
$$

Depending on the number of charged entities flowing from the solution to the electrode, we can write the amount of electricity $q$ as follows

$$
\begin{array}{rlrl} 
& N e=\int_{t 1}^{t 2} I . d t \\
\text { That is to say: } & z F n & =\int_{t 1}^{t 2} I . d t \\
\text { Knowing that: } & n & =\frac{m}{M}
\end{array}
$$

We then deduct the amount of aniline deposited on the electrode:

$$
m=\frac{M}{z F} \int_{t 1}^{t 2} I \cdot d t
$$

This allows us to determine the speed of the plating:

$$
\frac{d m}{d t}=\frac{M}{z F} I
$$

-Growth rate of the layer of the deposited conductive polymer:

Now determine the growth rate of the film deposited from the polyaniline on the anode:

We know that:

$$
m=\rho V
$$

Where $\rho$ denotes the density of the deposited polymer, $\mathrm{V}$ the volume of the layer deposited on the electrode.

So the volume speed of deposition is: 


$$
\frac{d V}{d t}=\frac{M}{z F \rho} I
$$

We have:

$$
\mathrm{V}=\mathrm{eS}
$$

If we identify $\mathrm{S}$ on the surface of the electrode we can then express the growth rate as follows:

$$
\frac{d e}{d t}=\frac{M}{z F \rho S} I
$$

This expression then presents the speed of formation of the film of the conductive polymer on the anode, so the thickness of the deposited layer has the form:

$$
e=\frac{M}{z F \rho S} \int_{t 1}^{t 2} I . d t
$$

En fonction de la quantité d'électricité $\mathrm{Q}$ on peut écrire :

$$
e=\frac{M Q}{z F \rho S}
$$

Hence :

$$
e=\frac{M}{z F \rho} \sigma_{S}
$$

The reaction of the electropolymerization can be followed, using electrochemical methods which include, chronoamperometry, cyclic voltammetry and impedimetry:

-Chronoamperometry:

Let's start with:

We can deduce the transient limit current from the diffusion equation of FICK which is expressed as follows:

$$
J=-D z F\left(\frac{\partial C}{\partial X}+\frac{\partial C}{\partial Y}\right)
$$

In vectorial notation, the diffusion current density is expressed as a function of the concentration gradient prevailing at the electrodeelectrolyte interface:

$$
\vec{J}=-D z F \vec{\nabla} C
$$

Now calculate the divergence of the current density vector:

$$
\vec{\nabla} \vec{J}=-D z F \Delta C
$$

This allows us to express the continuity equation linking the variation of the concentration as a function of time as well as its variation as a function of space:

$$
\begin{gathered}
-\frac{\partial C}{\partial t}=-D z F\left[\frac{\partial^{2} C}{\partial X^{2}}+\frac{\partial^{2} C}{\partial Y^{2}}+\frac{\partial^{2} C}{\partial Z^{2}}\right] \\
\frac{\partial C}{\partial t}-D z F\left[\frac{\partial^{2} C}{\partial X^{2}}+\frac{\partial^{2} C}{\partial Y^{2}}\right]=0
\end{gathered}
$$

Because the distribution of macro-ions satisfies the law of distribution of MAXWELLBOLTZMANN, the concentration can therefore be written:

$$
C=C_{0}\left[e^{\frac{z F \psi_{s}}{R T}}+e^{-\frac{z F \psi_{s}}{R T}}\right]
$$

And by introducing the thickness of the diffusion layer of NERNST $\delta_{\mathrm{D}}$, we can therefore express the diffusion current of the macro-ions from the diffusion layer towards the electrode by the equation below:

$$
J=z F D \frac{C}{\delta_{D}}=\frac{z F D}{\delta_{D C}} C_{0}\left[e^{\frac{z F \psi_{s}}{R T}}+e^{-\frac{z F \psi_{s}}{R T}}\right]
$$

By substituting $\delta_{\mathrm{D}}$ with its expression:

We find:

$$
\delta_{D}=\sqrt{D \tau_{D}}
$$

$$
J=z F D^{+1 / 2} \tau_{D}{ }^{-1 / 2} C
$$

This is the COTTRELL equation.

$$
\text { -Cyclic voltammetry: }
$$

Regarding cyclic voltammetry, the capacitive current depends on the capacity of the double layer as well as the scanning speed:

$$
I_{c}=S C_{d l} V_{B}
$$

If we explain the expression of $V_{B}$ :

$$
V_{B}=\frac{d E}{d t}
$$

Capacitive current takes the form:

$$
I_{C}=S C_{d l} \frac{d E}{d t}
$$

Which can be modeled by an equivalent derivative circuit, which allows us to write:

$U_{C}=\left[R_{\text {Electrode }}+R_{\text {Electrolyte }}+R_{\text {Diffusion }}+R_{t c}\right] I_{c} 38$

Therefore :

$$
U_{C(\text { sortie })}=S R C \frac{d E_{\text {entrée }}}{d t}
$$

Let us now express the faradaic current due to redox reactions:

$$
\begin{gathered}
I_{F}=I_{0}\left[e^{\frac{\alpha z F\left(E-E_{O x)}\right)}{R T}}-e^{-\frac{\beta z F\left(E-E_{r e d}\right)}{R T}}\right] \\
I_{F}=\frac{d Q}{d t}=z F \frac{d n}{d t}
\end{gathered}
$$

Depending on the concentration, we can express $I_{F}$ as follows:

$$
\begin{gathered}
I_{F}=z F V \frac{d C}{d t} \\
I_{F}=z F V k C\left[e^{-\frac{\alpha z F\left(E-E_{o x)}\right)}{R T}}-e^{-\frac{\beta z F\left(E-E_{r e d}\right)}{R T}}\right]
\end{gathered}
$$

Considering the reaction as a first order reaction:

$$
J_{F}=-z F \delta k C
$$

Under the theory of Erying's activated complex, the rate constant has the expression:

$$
k=\frac{K_{B} T}{2 \pi \hbar} e^{-\frac{\Delta G^{*}}{R T}} e^{\frac{\Delta S^{*}}{R}}
$$

$$
\begin{gathered}
J_{F}= \\
\frac{z F \delta K_{B} C T}{2 \pi \hbar} e^{\frac{\Delta S^{*}}{R}}\left[e^{-\frac{\left(\Delta G^{*}-\alpha z F\left(E-E_{O x}\right)\right)}{R T}}-e^{-\frac{\left(\Delta G^{*}+\beta z F\left(E-E_{r e d}\right)\right)}{R T}}\right]_{46}
\end{gathered}
$$

Let :

$$
A_{O x=}^{*} e^{\frac{\Delta S_{O x}^{*}}{R}} A_{R e ́ d}^{*}=e^{\frac{\Delta S_{r e d}^{*}}{R}}
$$



form:

In general, the density of faradic current has the

$$
\begin{gathered}
J_{F}= \\
\frac{z F \delta K_{B} C T}{2 \pi \hbar}\left[A_{o x}^{*} e^{-\frac{\left(\Delta G^{*}-\alpha z F\left(E-E_{o x}\right)\right)}{R T}}-A_{R e ́ d}^{*} e^{-\frac{\left(\Delta G^{*}+\beta z F\left(E-E_{r e d}\right)\right)}{R T}}\right]
\end{gathered}
$$

In such a way that the total current represents the total of the two capacitive and faradic currents:

$$
I=I_{C}+I_{F}
$$

At equilibrium the total current is zero, we can then express the concentration of ions in the immediate vicinity of the electrode as a function of the shear potential for low current densities as follows:

$$
\begin{array}{ll}
\text { follows: } & C_{m+}=C_{0 m+} e^{\frac{\alpha^{\prime} F \zeta}{R T}} \\
\text { Or : } \quad \zeta=-\frac{\sigma_{s}}{C_{d l}},
\end{array}
$$

$\sigma_{s}$ denotes the surface charge.

Application and experimental protocol of cyclic voltammetry

It is an electrochemical technique which makes it possible to locate the voltage of different reactions or elementary reaction stages and to determine their electrochemical characteristics. It consists in applying to the working electrode a voltage varying linearly over time, and recording the current response.

The applied voltage is:

$$
\begin{aligned}
& E=E_{i}+v_{B} t \\
& E=E_{\text {max }}-v_{B} t \\
& E=E_{\text {min }}+v_{B} t
\end{aligned}
$$

$E_{i}$ est la tension initiale, $v_{B}$ la vitesse de balayage en $\mathrm{V} / \mathrm{S}$ exprimer par :

$E_{i}$ is the initial voltage, $v_{B}$ the scanning speed in V / S expressed by:

$$
v_{B}=\frac{I_{c}}{S C_{d l}}=\frac{d E}{d t}
$$

$I_{c}$ : Capacitive current

S: Surface of the electrode

$\mathrm{C}_{\mathrm{d} l}$ : The capacity of the electrochemical double layer.

$$
C_{d l}=\frac{-\sigma_{s}}{\zeta}
$$

$\sigma_{\mathrm{s}}:$ Surface charge

$\zeta$ : Electrokinetic potential

In this technique, the electrode is stationary, so there is no convection of material on the surface of the electrode. Diffusion is therefore the only mode likely to transport electroactive species.

Migration is always ensured by a bottom salt or a support electrolyte thus ensuring an adequate conductivity.

The equation of the peak current as a function of the concentration of the electroactive species:

$$
I_{p}=\frac{K F S n^{\frac{3}{2}} D^{\frac{1}{2}} v_{B}^{\frac{1}{2}} C}{7^{\frac{1}{2}}}
$$

Randles-Sevick's equa potential $\zeta$

D: Diffusion coefficient of the electroactive species

S: Surface of the electrode

$\mathrm{n}$ : number of electrons involved

$\mathrm{C}$ : The concentration of the electroactive species

Taking into account the capacity of the electric double layer, the peak equation takes the form:

$$
I p=\zeta^{\frac{1}{2}} C_{d l} S n^{\frac{3}{2}} D^{\frac{1}{2}} v_{B}^{\frac{1}{2}} C
$$

Reversible and almost reversible system soluble product:

$$
\begin{gathered}
I p=4.631 \times 10^{6} n^{\frac{3}{2}}\left(D v_{B}\right)^{\frac{1}{2}} S C \\
E p=E_{p 1 / 2}-9.56 \times 10^{-4} \frac{T}{n}
\end{gathered}
$$

Half-wave potential

$$
\begin{gathered}
E p=E_{1 / 2}-\frac{R T}{n F} \\
E^{0}=\frac{E p_{a}+E p_{c}}{2} \\
E p_{a}-E p_{c}=2.3 \frac{R T}{n F} \\
\frac{I p_{a}}{I p_{G}}=1
\end{gathered}
$$

Stable product reversible system

Insoluble product

$$
\begin{aligned}
& I p=6.34 \times 10^{-6} n^{\frac{3}{2}}\left(\frac{D v_{B}}{T}\right)^{1 / 2} S C \\
& E p=E i-7.32 \times 10^{-5} \frac{T}{n} \\
& E p-E p_{1 / 2}=-6.64 \times 10^{-5} \frac{T}{n}
\end{aligned}
$$

Interests: Study of successive or simultaneous reactions; chemical reactions associated with electrochemical reactions.

-Determine the physical state at the interface of electroactive species

-Determination of E0 of redox couples

-Dosing and characterization

-Determination of energy differences between the valence bands and the semiconductor conduction bands.

For the assay: for low concentrations $\mathrm{C}<$ $10^{-5} \mathrm{~mol} / \mathrm{l}$, scanning is carried out at high speed.

The thickness of the solution in which the concentration variation occurs is a function of time.

$$
\delta=\mathrm{f}(\mathrm{t})
$$

When the sweep occurs one or more times "single sweep" or "cyclic sweep".

Monitoring of electropolymerization and electroplating reactions, we relates the number and 
height of the peaks observed to the number and thickness of the polymer films deposited.

\section{CONCLUSIONS}

The electrical conductivity of organic materials and especially macro-molecules, still remains an ample field of applications, rich and far from being exhausted, by virtue of the physical phenomena involved ensuring this conductivity (electron, hole, excitons, polarons, skyrmions, ....) as well as the still conceivable technological applications, from organic electronics to photovoltaics, up to the most spectacular fields of excitonic condensates, nanoelectrochemistry, skyrmionics, nuclear electrochemistry, biomolecular imaging and the quantum computer. But considerable difficulties still arise in front of the proliferation of these new materials, it is the control of their morphology on a nanoscopic scale during their synthesis, this morphology conditions all their properties, electrical, optical, electrochemical and even magnetic, this control largely depends on the physico-chemical methods used for the synthesis and characterization of these macro-molecules as well as a deep understanding of the links existing between the different mechanisms of electrical conduction of these materials and their electronic structures, their redox properties as well as their molecular masses, their organizations and their stereochemistry.

\section{REFERENCES}

[1] Pradyumna Mogre, Sharanabasava V. Ganachari, Jayachandra S. Yaradoddi, Nagaraj N. Banapurmath, Anand M. Hunashyal, Ashok S. Shettar, Synthesis and characterization studies of polyaniline nano fibres. Advanced Materials Proceedings, 2018, 3(3), 178-180.

[2] N. Gospodinova, L. Terlemezyan, Conducting Polymers Prepared by Oxidative, Polymerization: Polyaniline, Prog. Polym. Sci, , Vol. 23, 1443-1484, 1998
[3] Pradyumna Mogre, Sharanabasava V. Ganachari, Jayachandra S. Yaradoddi, Nagaraj N. Banapurmath, Anand M. Hunashyal, Ashok S. Shettar, Synthesis and characterization studies of polyaniline nano fibres. Advanced Materials Proceedings, 2018, 3(3), 178-180.

[4] Ateh DD, Navsaria HA, Vadgama P. Polypyrrole-based conducting polymers and interactions with biological tissues. J R Soc Interface 2006;3:741-52

[5] Shirakawa H, Louis EJ, MacDiarmid AG, Chiang CK, Heeger AJ. Synthesis of electrically conducting organic polymers: halogen derivatives of polyacetylene, $(\mathrm{CH}) x$. J Chem Soc Chem Commun 1977:578-80.

[6] Zhou DD, Cui XT, Hines A, Greenberg RJ. Conducting polymers in neural stimulation applications. In: Zhou DD, Greenbaum E, editors. Implantable neural prostheses, vol. 2. Berlin: Springer; 2010. p. 217-52.

[7] Lakard B, Ploux L, Anselme K, Lallemand F, Lakard S, Nardin M, et al. Effect of ultrasounds on the electrochemical synthesis of polypyrrole, application to the adhesion and growth of biological cells. Bioelectrochemistry 2009;75:148-57 .

[8] Kotwal A, Schmidt CE. Electrical stimulation alters protein adsorption and nerve cell interactions with electrically conducting biomaterials. Biomaterials 2001;22:1055-64.

[9] Wallace GG, Smyth M, Zhao H. Conducting electroactive polymer-based biosensors. Trends Analyt Chem 1999;18:245-51.

[10] Vivekanandan J, Ponnusamy V, Mahudeswaran A, Vijayanand PS. Synthesis, characterization and conductivity study of polyaniline prepared by chemical oxidative and electrochemical methods. Arch.Appl. Sci. Res. 2011;3(6):147-53.

[11] Ninh DH, Thao TT, Long PD, Dinh NN. Characterization of Electrochromic Properties of

Polyaniline Thin Films Electropolymerized in H2SO4 Solution. Open J. Org. Polym. Mater. 2016 Jan 7;6(01):30.

[12] Bavane RG. Synthesis and Characterization of thin Films of Conducting Polymers for Gas Sensing Application. SOPS.NMU: Jalgaon;2014 chapter three,Synthesis of polyaniline (PANI); p.22-37.

[13] H. Tsutumi, S. Yamashita, T. Oishi, "Preparation of polyan i-line -poly (pstyrenesulfonic acid) composite by post polymerization and application as positive active 
m a-terial for a rechargeable lithium battery," Journal of Applied Electrochemistry, vol. 27, pp. 477-481, June (1997).

[14] Han MG, Lee YJ, Byun SW and Soon Im S. 2001. Physical properties and thermal transition of polyaniline film. Synthetic Met , 124, pp:337343.

[15] Ayad M.M and Zaki EA. 2008. Doping of polyaniline films with organic sulfonic acids in aqueous media and the effect of water on these doped films, Eur.Polym. J., 44, pp:3741-3747.

[16] Sniechowski M, Djurado D, Dufour B, Rannou P and Pron A. 2004. Direct analysis of lamellar structure in polyaniline protonated with plasticizing dopants, Synthetic Met, 143, pp:163169.

[17] Sniechowski M, Djurado D, Dufour B, Rannou P and Pron A. 2004. Direct analysis of lamellar structure in polyaniline protonated with plasticizing dopants, Synthetic Met, 143, pp:163169.

[18] Ayad M.M and Zaki EA. 2008. Doping of polyaniline films with organic sulfonic acids in aqueous media and the effect of water on these doped films, Eur.Polym. J., 44, pp:3741-3747.

[19] Jiansheng Wua, Yimeng Sunb, Wei Xub, Qichun Zhanga. 2014. Investigating thermoelectric

properties of doped polyaniline nanowires , Synthetic Metals, 189, pp:177-182.

[20] Pornputtkul Y, Kane-Maguire L.A.P and Wallace G.G.2006. Influence of electrochemical polymerization temperature on the optical properties of(+)-camphor sulfonic acid-doped polyaniline , Macromolecules, 39, pp:5604-5610 .

[21] Khanna P.K, Singh N, Charan S and Viswanath A.K. 2005. Synthesis of $\mathrm{Ag} /$ polyanilinenano composite via aninsituphotoredox mechanism, Mater. Chem. Phys., 92, pp:214-219.

[22] Hussain A. and Kumar A. 2000. Electrochemical synthesis and characterization of chloride doped polyaniline, Bulletin of Material Science, 26(3), pp:329-334 .

[23] Kargirwar, S.R.; Thakare, S.R.; Choudhary, M.D.; Kondawar, S.B.; Dhakate, S.R.; Adv. Mat. Lett. 2011, 2(6), 397.

[24] Kushwah, B.S.; Upadhaya, S.C.; Shukla, S.; Sikarwar, A.S.; Sengar, R.M.S.; Bhadauria, S. Adv. Mat. Lett. 2010, 2(1), 43.

[25] Tiwari, A.; Kumar, R.; Prabhakaran, M.; Pandey, R.R.; Kumari, P.; Chadurvedi, A.;
Mishra, A.K. Polymers for Advanced Technologies 2010, 21, 615.

[26] Tiwari, A.; Sen, V.; Dhakate, S.R.; Mishra, A.P; Singh, V. Polymers for Advanced Technologies, 2008, 19, 909.

[27] Tiwari, A. Journal of Polymer Research 2008, 15(4), 337.

[28] Tiwari, A. Journal of Macromolecular Science, Part A: Pure and Applied Chemistry, 2007, 44(7), 735.

[29]. Kondawar, S.B.; Thakare, S.R.; Bompilwar, S.; Khati, V. Int. J. Mod. Phys. B, 2009, 23 (15), 3297.

[30] Tiwari, A.; Prabaharan, M.; Pandey, R.; Li, S. Journal of Inorganic and Organometallic Polymers and Materials, 2010, 20(2), 380.

[31] Kondawar, S.B.; Acharya, S.A.; Dhakate, S.R. Adv. Mat. Lett. 2011, 2(5), 362.

[32] Limin L, Enhui L, Yanjing Y, Haijie Sh, Zhengzheng H, Xiaoxia X (2010) Nitrogen containing carbons prepared from polyaniline as anode materials for lithium secondary batteries. Mater Lett 64:2115-2117

[33] Chen Y, Yang G, Zhang Z, Yang X, Hou W, Zhu J (2010) Polyaniline intercalated layered vanadium oxide nanocomposites onepot hydrothermal synthesis and application in lithium battery. Nanoscale 2:2131-2138

[34] Tsumura A, Koezuka H, Ando T (1986) Macromolecular elec-tronic device: field effect transistor with a polythiophene thin film. Appl Phys Lett 49:1210-1217

[35] Tiwari, A.; Sen, V.; Dhakate, S.R.; Mishra, A.P; Singh, V. Polymers for Advanced Technologies, 2008, 19, 909. 